absorption into nanotubes. In the case of tungsten disulfide, the crystal symmetry of the nanotube is reduced with respect to that of the monolayer and bilayer, because of the tube's curved walls. The combination of excellent light absorption and low crystal symmetry means that the nanotube exhibits a substantial BPVE. The density of the electric current associated with the BPVE surpasses that of the materials that have inherently low symmetry, even though the conversion efficiency of the BPVE is still much lower than that of the junction-based photovoltaic effect in conventional solar cells.

The authors' results demonstrate the great potential of nanotubes in harvesting solar energy, and raise several technological challenges and scientific questions. From an applications perspective, it would be instructive to fabricate and characterize a solar cell that is made up of an array of semiconductor nanotubes, to check the feasibility of scaling up the approach. The direction of the current generated by the BPVE in each nanotube would be largely determined by the material's internal symmetry. Therefore, a uniform symmetry across the nanotube array would be needed to gather a collective current from the solar cell. In a worst-case scenario, if the currents generated in different nanotubes were in opposite directions, they would cancel each other out.

An important but unanswered question is whether the BPVE and the junction-based photovoltaic effect could cooperate in the same solar cell, to boost the overall efficiency. These two effects could harness solar energy in a successive manner. Nevertheless, despite the remaining challenges, Zhang and colleagues' work provides a possible route towards the design of highly efficient, unconventional solar cells. .

Ming-Min Yang and Marin Alexe are in the Department of Physics, University of Warwick, Coventry CV4 7AL, UK.

e-mails:mingmin.yang.1@warwick.ac.uk; m.alexe@warwick.ac.uk

1. Zhang, Y. J. et al. Nature 570, 349-353 (2019).

2. Chynoweth, A. G. Phys. Rev. 102, 705-714 (1956).

3. Chaplin, D. M., Fuller, C. S. \& Pearson, G. L. J. Appl. Phys. 25, 676-677 (1954).

4. Fridkin, V. M. Photoferroelectrics (Springer, 1979).

5. Grinberg, l. et al. Nature 503, 509-512 (2013).

6. Yang, M. M., Kim, D. J. \& Alexe, M. Science $\mathbf{3 6 0}$, 904-907 (2018).

\section{Sex chromosomes manipulate mate choice}

Female mate choice in some species selects for traits that are harmful to males. A hypothesis to explain how such mating preferences might evolve puts the spotlight on sex chromosomes. SEE LETTER P.376 that affect mating preferences have a range of effects on other traits. This is the most probable explanation for why females of some species have pre-existing preferences for traits that don't exist in males of their own species. For example, males of some species of swordtailed fishes have tails with greatly elongated lower fin rays. Females from a related species in which males do not have this 'sword' structure nevertheless have a preference for it, as assessed by their attraction to males of their own species that have had an artificial sword surgically attached to them ${ }^{3}$.

The second kind of hypothesis rests instead on indirect selection. Genetic variation in genes that affect mating preferences becomes correlated with genetic variation for other traits, and selection acting directly on the latter
A mong some of the most spectacular sights in nature are the bizarre mating displays of certain animals. These displays risk decreasing male survival because the bright colours and loud calls of the males might attract predators (Fig. 1). Yet despite this risk of harm, these traits have nevertheless evolved because only the males that can make the most exaggerated displays are chosen by females as mates. An enduring mystery in evolutionary biology is why females of these species have evolved such mating preferences. On page 376 , Muralidhar ${ }^{1}$ uses a population-genetics model to show how these types of preference can evolve if the versions of genes that contribute to the male mating display have beneficial effects when those versions of genes are present in females.

A variety of hypotheses for how female mating preferences evolve have been inspired by results from fieldwork, experiments and mathematical modelling. Most of these hypotheses fall into one of two main camps ${ }^{2}$. In the first, mating preferences evolve by direct selection - the genes that affect mating preferences are themselves direct targets of selection. For example, if some males offer superior parental care, female mating preferences for choosing those males will spread by natural selection. This is why females in monogamous species often prefer males that are not brightly coloured and that are therefore more likely to evade predators and survive to help rear offspring.

Direct selection can also act when genes

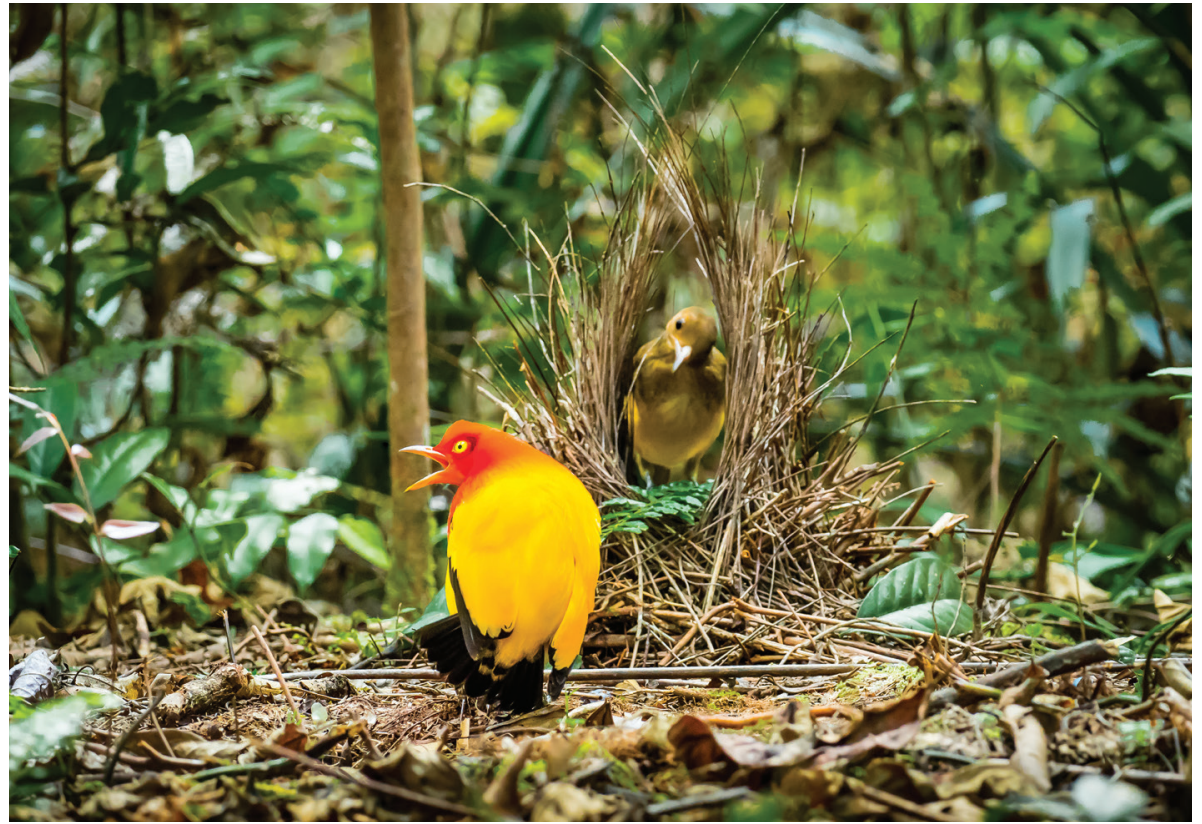

Figure 1 | A colourful mating display. A vibrantly coloured male flame bowerbird (Sericulus aureus) engages in a mating display as a female of the species looks on. The male's bright colours make the bird easy for predators to spot, raising the conundrum of how female mating preferences evolve for traits that are harmful to males. Muralidhar ${ }^{1}$ puts forward a hypothesis for how such mating preferences might arise. Whether this idea could explain the bowerbird case is unknown. 
can cause mating preferences to evolve as a side effect. One example of this is what is called the good-genes hypothesis. In this model, for example, versions of genes that boost the immune system lead to better health, which enables the males that carry these versions to be more brightly coloured. The females that have mating preferences for the brightest males will pass these good genes, which improve survival, to their offspring, along with the versions of genes for a mating preference for bright males. As selection causes these beneficial immune-system genes to spread, they also drag along the genes that drive the preference for brighter males. This hypothesis is popular among behavioural ecologists, but the evidence in support of it is mixed ${ }^{4}$.

The hypothesis put forward by Muralidhar to explain the evolution of female mating preferences is also based on indirect selection. This model hinges on sexually antagonistic selection - the situation in which a version of a gene that is beneficial when present in one sex is harmful when present in the other sex. Among evolutionary geneticists, there is a growing appreciation of the prevalence of sexually antagonistic selection and its myriad consequences ${ }^{5}$. Imagine a genetic mutation that causes an increase in the redness of both male and female plumage. This mutation might increase male fitness by increasing mating success, but it would decrease female fitness by increasing predation. Therefore, genes that cause females to prefer to mate with redder males will cause their sons from such matings to have high fitness, whereas their daughters would have low fitness. Those two opposing effects on fitness at the population level would offset each other, and, on balance, mating preferences for redder males would be selected neither for nor against.

But could there be an exception to this logic if genes that affect mating preference are inherited in an unusual way? Consider what might happen if such genes are on sex chromosomes.

In mammals and fruit flies, males have two different sex chromosomes (X and Y), whereas females have two X chromosomes. Organisms such as birds and butterflies ${ }^{6}$ have sex chromosomes called $\mathrm{Z}$ and $\mathrm{W}$, and the opposite arrangement exists - the males have two of the same type of sex chromosome ( $\mathrm{Z}$ chromosome), whereas the females have one $\mathrm{Z}$ and one $\mathrm{W}$ chromosome. A gene that affects mating preference and is located on a $\mathrm{W}$ chromosome exists only in females, and never in males. Therefore, a female that has a version of a gene on the $\mathrm{W}$ chromosome that causes a mating preference for a trait that decreases male survival will pass that version only to her daughters. If those daughters also receive from their fathers the versions of genes for a trait that is beneficial to females, the daughters will have high fitness, and this W chromosome will spread. These nefarious chromosomes can be described as selfish - they spread regardless of their effect on male fitness.

Muralidhar's mathematical analysis shows that genes affecting mating preference that are carried by other types of sex chromosome (X, Y and Z) also have evolutionary dynamics that differ from those of genes that affect mating preference but are carried on non-sex chromosomes. But of the four types of sex chromosome, Muralidhar found that it is the W chromosome that has the greatest potential to favour the spread of versions of genes that increase female mating preferences for exaggerated (and harmful) male traits.

Models show what is possible, but only data can reveal whether the possible is a reality. Is there evidence that sex chromosomes harbour genes that affect mating preferences? Muralidhar reviewed the genetics of mating preferences that have been reported for 36 species. In more than half of these species, there is indeed evidence that genes that affect mating preferences are carried on the sex chromosomes. Disappointingly, none of these preferences is linked to the $\mathrm{W}$ chromosome, but the number of existing studies available for this analysis is small.

A second opportunity to bring data to bear on this model relates to its prediction that species that have ZW chromosomes should be more prone to evolve female mating preferences for sexually antagonistic traits than those with XY chromosomes. Indeed, some researchers have concluded that species that have ZW sex chromosomes tend to have exaggerated sexual displays more often than do those with XY chromosomes ${ }^{7}$. Muralidhar's work provides a call for more comparative data on the inheritance of mating preferences and the connections between sex-determination systems and sexual ornamentation.

Could this selfish-sex-chromosome hypothesis explain female mating preferences for vibrant colours, as is the case for the brilliantly coloured male flame bowerbirds (Sericulus aureus), which are preferred by the dull-coloured females of that species (Fig. 1)? Perhaps not. Genes that affect colour (and most other traits) tend to have similar effects on both sexes. Thus, females that choose dull-coloured males will have dull-coloured daughters that will survive well. If genes that affect mating preferences are carried on the $\mathrm{W}$ chromosome, this would favour the evolution of preferences for dull males.

It seems that some other explanation might be needed for cases such as that of the flame bowerbird. It could well turn out that preferences for different types of male trait evolve by different evolutionary pathways. If so, the decades-old debates over which hypotheses best explain how mating preferences evolve ${ }^{2}$ might ultimately transform into discussions of which mechanisms operate most commonly in certain contexts. -

Mark Kirkpatrick is in the Department of Integrative Biology, University of Texas, Austin, Texas 78712, USA.

e-mail:kirkp@mail.utexas.edu

1. Muralidhar, P. Nature 570, 376-379 (2019).

2. Kirkpatrick, M. \& Ryan, M. J. Nature $\mathbf{3 5 0}, 33-38$ (1991).

3. Basolo, A. L. Proc. R. Soc. B 259, 307-311 (1995).

4. Prokop, Z. M., Michalczyk, Ł., Drobniak, S. M., Herdegen, M. \& Radwan, J. Evolution 66. 2665-2673 (2012)

5. Rowe, L., Chenoweth, S. F. \& Agrawal, A. F. Am. Nat. 192, 274-286 (2018).

6. Bachtrog, D. et al. PLoS Biol. 12, e1001899 (2014).

7. Reeve, H. K. \& Pfennig, D. W. Proc. Natl Acad. Sci. USA 100, 1089-1094 (2003).

This article was published online on 5 June 2019.

\section{MATERIALS SCIENCE}

\section{Crazy colour}

The formation of microscopic pores and fibrils in polymers under stress $-\mathbf{a}$ process called crazing - often preludes material failure. Controlled crazing has now been used to produce an array of colours in polymer films. SEE LETTER P.363

\section{SEUNG HWAN KO}

W hen a typical transparent, glassy polymer is bent or stretched, partial whitening of the material often occurs just before it cracks or fractures ${ }^{1}$. This unpredictable phenomenon is called crazing, and has generally been seen as something to be avoided. But on page 363 , Ito et al. ${ }^{2}$ report that crazing can be fully controlled, and can be used to endow transparent polymers with colour. Controlled crazing could therefore be developed as the basis of an inkless, high-resolution method for printing colour on various flexible and transparent polymer materials.
Transparent polymers have conventionally been colorized by mixing them with pigments, or by printing pigment-containing ink on polymer surfaces. However, transparent polymers can also be colorized by producing microscopic structures within the materials - an effect known as structural coloration. Structural colours are frequently observed in nature, for example in butterfly wings ${ }^{3}$. Ito and co-workers use crazing as the basis for structural colour.

Crazing patterns in polymers form in a direction perpendicular to the applied stress, and consist of interpenetrating, micrometrescale voids bridged by highly oriented polymer 